\section{Response to "Acute kidney injury occurs only rarely in patients with Kawasaki disease"}

Advance online publication, 20 September 2017; doi:10.1038/pr.2017.192

To the Editor: The authors of "Letter to the Editor-Acute kidney injury occurs only rarely in patients with Kawasaki disease" stated that a similar study performed by them at a city general hospital in Japan showed a different prevalence of acute kidney injury (AKI) in Kawasaki disease (KD) patients than our study (1). They proposed that the difference in disease severity might affect the results and jumped to the conclusion that AKI occurs only rarely in patients with $\mathrm{KD}$. The objective evidence of different severity, according to them, was achieved by comparing coronary artery abnormality/lesion (CAL) rates. CALs include cases of coronary artery aneurysm (CAA) and milder cases with only coronary artery dilation/ectasia.

First, the authors mistook CAA rate for CAL rate in the large epidemiological study in Taiwan (2). The large epidemiological study in Taiwan that the authors quoted showed a CAA rate (which was not the overall CAL rate, as they mistook) of $7.2 \%$ among KD patients (2). Our study revealed a similar CAA rate of $6.3 \%$, but the overall CAL rate was $21.7 \%$. A study done at the Toronto Hospital for Sick Children with $1,374 \mathrm{KD}$ patients showed a CAA rate of $6 \%$, and, along with coronary artery ectasia cases, the CAL rate was $19 \%$ (3). Second, it has previously been claimed that the CAL rate in $\mathrm{KD}$ patients is lower in Japan than in the other countries. However, this only reflects incongruence in the case definition between the American Heart Association (AHA) and the Japanese Circulation Society (JCS). After carefully reviewing KD patients from centers in the United States and Japan by standardizing the coronary artery diameter with $Z$ score for comparison, the Japanese subjects actually had a higher rate of CAL (4). If the CAL definition is made solely according to the JCS criteria without assessing the coronary artery diameter $Z$-score, their study results will not be able to be compared with the results obtained from other countries including Taiwan, which adopts the AHA criteria.

Moreover, renal involvement in $\mathrm{KD}$ is not uncommon, at least in Taiwanese patients, as a previous study had already shown high incidence of renal inflammation by DMSA renal SPECT (5), and our study showed AKI, defined as a patient's serum creatinine level higher than 1.5 times the upper limits of age-specific serum creatinine levels (1). We are afraid that their criteria for AKI were different from ours; thus, the two results could not be compared with each other.

In summary, there was no obvious disease severity difference among our study, the nationwide study in Taiwan, and those conducted in other countries with regard to CAL rate. We suggest routine evaluation of the renal function for every patient with KD. Further studies are needed to explore the difference of $\mathrm{AKI}$ incidence among $\mathrm{KD}$ patients from different populations or $\mathrm{KD}$ patients with different disease severity.

\section{STATEMENT OF FINANCIAL SUPPORT}

No financial assistance was received and there were neither financial ties to products nor potential/perceived conflicts of interest in the study.

Disclosure: The authors declare no conflict of interest.

\section{Gwo-Tsann Chuang ${ }^{1}$ and Luan-Yin Chang ${ }^{1}$}

${ }^{1}$ Department of Pediatrics, National Taiwan University Hospital, College of Medicine, National Taiwan University, Taipei, Taiwan.

\section{Correspondence: Luan-Yin Chang (lychang@ntu.edu.tw)}

\section{REFERENCES}

1. Chuang GT, Tsai IJ, Lin MT, Chang LY. Acute kidney injury in patients with Kawasaki disease. Pediatr Res 2016;80:224-7.

2. Lin MC, Lai MS, Jan SL, Fu YC. Epidemiologic features of Kawasaki disease in acute stages in Taiwan, 1997-2010: effect of different case definitions in claims data analysis. J Chin Med Assoc 2015;78:121-6.

3. Sabharwal T, Manlhiot C, Benseler SM, et al. Comparison of factors associated with coronary artery dilation only versus coronary artery aneurysms in patients with Kawasaki disease. Am J Cardiol 2009;104: $1743-7$.

4. Ogata S, Tremoulet AH, Sato Y, et al. Coronary artery outcomes among children with Kawasaki disease in the United States and Japan. Int J Cardiol 2013;168:3825-8.

5. Wang JN, Chiou YY, Chiu NT, Chen MJ, Lee BF, Wu JM. Renal scarring sequelae in childhood Kawasaki disease. Pediatr Nephrol 2007;22:684-9. 